\section{Exercises for improving bone strength}

\section{H Turner, A G Robling} The effectiveness of exercise on bone strength depends on its type,
timing, and duration

$\mathrm{R}$ egular exercise has effects on bone density, size, and shape, resulting substantial improvements in mechanical strength. The positive association between exercise and bone mass has prompted many physicians and public health officials to recommend that people engage in daily exercise, with the goal of reducing the incidence of osteoporotic fracture and the morbidity/mortality that ensues. However, there is no clear consensus on exactly how one should exercise in order to reap the greatest returns in terms of bone health. What exercises are best? How often should one exercise? Is it sometimes better not to exercise?

\section{A LITTLE BONE GOES A LONG WAY}

Proper exercise can add new bone and/ or reduce bone loss to ultimately affect bone mass, but bone mass (or areal bone mineral density) is merely a surrogate measure for bone strength. The real issue at hand is whether or not a person will fracture their hip, spine, or wrist. Bone mineral content and bone mineral density are related to bone strength, but sometimes inferring strength from bone mineral measurements can be misleading. With exercise, small gains in bone mineral can result in large improvements in bone strength, because new bone formation is often localised to bone surfaces where mechanical strains (stresses) were greatest.

\section{NOT ALL EXERCISES ARE EQUALLY EFFECTIVE}

The pioneering studies of Hert et $a l^{2}$ performed over 30 years ago showed that bone tissue responds to dynamic rather than static loading. This finding provides important information about how bone cells detect mechanical loading. As static loads (even those that produce fairly large stresses or strains) do not initiate osteogenesis, stress or strain in the tissue cannot by itself be the primary stimulus for cellular response. Instead there must be something special about dynamic loadingfor instance, dynamic loading creates fluid movement in bone's lacunar-canalicular network, which in turn generates shear stresses on the plasma membranes of resident osteocytes, bone lining cells, and osteoblasts. Bone cells are highly sensitive to fluid shear stresses, and respond by initiating a cascade of cellular events. High impact exercises that probone matrix best drive fluid through the lacunar-canalicular network system. Thus increasing loading rate is one step toward more effective application of mechanical forces to promote osteogenesis.

\section{PROLONGED EXERCISE HAS DIMINISHING RETURNS}

Bone cells become desensitised to prolonged mechanical stimulation. Notably Umemura $\mathrm{et} \mathrm{al}^{3}$ showed that rats trained to jump many times a day increased the anabolic response became saturated after about 40 loading cycles; animals trained to jump 100 times a day did not improve their bone mass significantly over those trained to jump 40 times a day. The data from Umemura et al fit almost perfectly a logarithmic relationship, indicating that bone loses over 95\% of its mechanosensitivity after only 20 loading cycles. Presumably bone cell mechanosensitivity will return after a period of no loading. This concept was tested by Robling et al ${ }^{4}$ in an experiment in which rats were subjected to mediolateral bending of the tibia. All six groups of rats in the experiment received 360 cycles a day of the same loading stimulus, delivered as 90 continuous cycles, four times a day. Some of the rats were allotted eight hours between each of the four daily bouts, others were given $4,2,1,0.5$, or 0 hours of rest between each of the four daily bouts. Load induced bone formation was improved by the rest periods, and as the rest (no loading) periods were lengthened, bone formation was enhanced further. With a rest period of four hours between loading bouts, loading induced bone formation was almost doubled. After 24 hours of rest, $98 \%$ of bone mechanosensitivity returns. Consequently the osteogenic response to exercise can be enhanced by regimens duce large rates of deformation of the mass of their femora and tibiae, but the that incorporate periods of rest between short vigorous skeletal loading sessions.

\section{ASSESSING EXERCISE: THE OSTEOGENIC INDEX}

Bone formation with exercise can be estimated using an osteogenic index (OI). The OI depends on the exercise intensity and degree of desensitisation. Laboratory data suggest that exercise intensity should be calculated as peak magnitude of load (or stress) multiplied by the loading frequency. ${ }^{5}$ Translating the laboratory data in terms of human exercise suggests that the rate of increase of the ground reaction force will best estimate the skeletal response. The OI for multiple bouts of loading depends on the recovery time allowed between sessions. The osteogenic potential of exercise can be increased when the daily exercise is divided into two shorter sessions separated by eight hours. For example, consider 120 jumps a day performed in one session or broken into either two sessions of 60 jumps separated by eight hours or three sessions of 40 jumps separated by four hours. Breaking 120 jumps into two sessions improves the OI by almost $50 \%$ but dividing the day further into three sessions four hours apart does not further improve the OI. ${ }^{6}$ If one wishes to reduce exercise time, it is far better to shorten each exercise session than to reduce the number of sessions.

\section{START EARLY TO PREVENT OSTEOPOROSIS}

Although exercise has clear benefits for the skeleton, engaging in exercise during skeletal growth is unequivocally more osteogenic than exercise during skeletal maturity. ${ }^{7}$ The biological mechanisms for this phenomenon are not yet fully understood but are probably related to the fact that, during growth, the bone surfaces are covered with a greater proportion of active osteoblasts than after skeletal maturity. Periosteal expansion occurs predominantly during growth, and consequently the childhood and adolescent years provide a window of opportunity to significantly enhance periosteal growth with exercise. Periosteal growth determines the periosteal breadth of a bone, which is important in skeletal health for two main reasons. Firstly, addition of bone to the periosteal surface improves the bending and torsional strength of the bone most effectively. Secondly, resorption of bone from the periosteal surface is extremely rare in the adult; usually it is the trabecular, endocortical, and Haversian bone surfaces that undergo remodelling. These observations indicate that the periosteal breadth of a bone will remain intact 
until senescence. Consequently, vigorous exercise during growth and young adulthood may well reduce fracture risk in later decades. ${ }^{8}$

Br J Sports Med 2005;39:188-189.

doi: 10.1136/bjsm.2004.016923

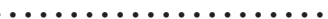

Authors' affiliations

C H Turner, Department of Biomedical Engineering, Indiana University, Purdue University Indianapolis, Indianapolis, IN 46202, USA

A G Robling, Department of Anatomy \& Cell Biology, Indiana University, Indianapolis, IN 46202
Correspondence to: Dr Turner, Department of Biomedical Engineering, 1120 South Drive, FH 115, Indianapolis, IN 46202, USA; turnerch@ iupui.edu

Competing interests: none declared

\section{REFERENCES}

1 Robling AG, Hinant FM, Burr DB, et al. Improved bone structure and strength after long-term mechanical loading is greatest if loading is separated into short bouts.

J Bone Miner Res 2002:17: 1545-54.

2 Hert J, Lisková M, Landa J. Reaction of bone to mechanical stimuli. 1. Continuous and intermittent loading of tibia in rabbit. Folia Morphol (Praha) $1971 ; 19: 290-300$.
3 Umemura Y, Ishiko T, Yamauchi T, et al. Five jumps per day increase bone mass and breaking force in rats. J Bone Miner Res 1997:12:1480-5.

4 Robling AG, Burr DB, Turner CH. Recovery periods restore mechanosensitivity to dynamically loaded bone. J Exp Biol 2001;204(Pt 19): 3389-99.

5 Turner $\mathrm{CH}$. Three rules for bone adaptation to mechanical stimuli. Bone 1998;23:399-407.

6 Turner CH, Robling AG. Designing exercise regimens to increase bone strength. Exerc Sport Sci Rev 2003;31:45-50.

7 Kannus $\mathbf{P}$, Haapasalo $H$, Sankelo $M$, et al. Effect of starting age of physical activity on bone mass in the dominant arm of tennis and squash players. Ann Intern Med 1995; 123:27-31.

8 Karlsson MK, Ahlborg $\mathrm{H}$, Obrant KJ, et al. Exercise during growth and young adulthood is associated with reduced fracture risk in old ages. J Bone Miner Res 2002;17(suppl 1):S297.

\section{ELECTRONIC PAGES}

\section{Online original articles}

I

he following electronic only articles are published in conjunction with this issue of BJSM (see also pages 211

and 216)

\section{Neck injuries presenting to emergency departments in the United States from 1990 to 1999 for ice hockey, soccer, and American football \\ J S Delaney, A Al-Kashmiri}

Objective: To examine the number and rate of neck injuries in the community as a whole for ice hockey, soccer, and American football by analysing data from patients presenting to emergency departments in the United States from 1990 to 1999.

Methods: Data compiled for the US Consumer Product Safety Commission were used to generate estimates for the total number of neck injuries and the more specific diagnoses of neck fractures, dislocations, contusions, sprains, strains, and lacerations occurring nationally from 1990 to 1999. These data were combined with yearly participation figures to generate rates of injury presenting to emergency departments for each sport.

Results: There were an estimated 5038 neck injuries from ice hockey, 19341 from soccer, and 114706 from American football. These could be broken down as follows: 4964 contusions, sprains, or strains from ice hockey, 17927 from soccer, and 104483 from football; 105 neck fractures or dislocations from ice hockey, 214 from soccer, and 1588 from football; 199 neck lacerations for ice hockey, 0 for soccer, and 621 for football. The rates for total neck injuries and combined neck contusions, sprains, or strains were higher for football than for ice hockey or soccer in all years for which data were available.

Conclusion: The rate of neck injury in the United States was higher in football than in ice hockey or soccer in the time period studied.

(Br J Sports Med 2005;39:e21) http://bjsm.bmjjournals.com/ cgi/content/full/39/1/e21

\section{Methods for injury surveillance in international cricket J W Orchard, D Newman, R Stretch, et al}

Background: The varying methods of cricket injury surveillance have made direct comparison of published studies in this field impossible.
Methods: A consensus regarding definitions and methods to calculate injury rates in cricket was sought between researchers in this field. This was arrived at through a variety of face to face meetings, email communication, and draft reviews between researchers from six of the major cricket playing nations.

Results: It is recommended that a cricket injury is defined as any injury or other medical condition that either (a) prevents a player from being fully available for selection for a major match or (b) during a major match, causes a player to be unable to bat, bowl, or keep wicket when required by either the rules or the team's captain. Recommended definitions for injury incidence (for matches, training sessions, and seasons) and injury prevalence are also provided. It is proposed that match injury incidence is calculated using a denominator based on a standard time estimated for player exposure in matches, for the purposes of simplicity. This will allow all injury surveillance systems, including those with limited resources, to make calculations according to a standard definition.

Conclusion: The consensus statement presented provides a standard which, if followed, allows meaningful comparison of injury surveillance data from different countries and time periods, which will assist in the possible identification of risk factors for injury in cricket.

"Methods for injury surveillance in international cricket", a collaboration between cricket authorities in Australia, the United Kingdom, South Africa, New Zealand, the West Indies, and India, is being published simultaneously in the Journal of Science and Medicine in Sport, the British Journal of Sports Medicine, the South African Journal of Sports Medicine, and the New Zealand Journal of Sports Medicine. The paper was concurrently reviewed and edited by the Journal of Science and Medicine in Sport and the British Journal of Sports Medicine.

(Br J Sports Med 2005;39:e22) http://bjsm.bmjjournals.com/ cgi/content/full/39/1/e22 